\title{
Le concept de proximité comme source de différenciation : proposition d'une grille de lecture des positionnements voulus des distributeurs français
}

\section{Claire Capo}

Aix-Marseille Université, CRET-LOG, France

claire.capo@univ-amu.fr

\section{Odile Chanut}

Aix-Marseille Université, CRET-LOG, France

odile.chanut@univ-amu.fr

La distribution alimentaire en France fait face à des changements de société et des attentes accrues des consommateurs : plus de services, plus de praticité, moins de déplacements... Si l'hypermarché s'essouffle, le commerce de proximité connaît un renouveau avec le lancement récent de nouveaux concepts de distribution sous enseignes. L'article propose une grille de lecture analytique des positionnements voulus de ces nouveaux concepts déclinant sept dimensions opérationnalisées de la proximité: proximités spatiale, fonctionnelle, relationnelle, identitaire, de processus, inter-organisationnelle et prix. La grille est utilisée ensuite pour identifier les points communs aux enseignes de proximité -une structure de coûts et une logistique compliquées, la franchise, les MDD (marques de distributeur)- et pour distinguer, à titre d'illustration, les spécificités des positionnements voulus de trois enseignes -U Express, Monoprix et Carrefour City Café. Il conclut sur l'idée que la grille de lecture proposée peut être utilisée au-delà des nouveaux concepts de proximité pour analyser les positionnements d'autres formats, tels que l'hypermarché ou le drive.

Mots clés : Proximité, Commerce de proximité, Positionnement voulu, Convenience, Grille de lecture.

\section{Introduction}

«Le commerce de proximité a de l'avenir » titrait L'express dans un article publié le 22 mars $2012^{1}$; et d'ajouter: «Les nouveaux modes de vie et de consommation redonnent de

\footnotetext{
${ }^{1}$ http://www.lexpress.fr/emploi-carriere/franchise/le-commerce-de-proximite-a-de-l-avenir 1097033.htmI consulté le 20 octobre 2012.
} 
l'attractivité aux commerces de centre-ville. Attentes et tendances dont les enseignes peuvent tirer parti ». Le phénomène touche en particulier le commerce alimentaire. En effet, on observe récemment en France un renouveau des formats de vente «du coin de la rue » avec des enseignes comme Daily Monop', Carrefour City, Casino Shopping ou encore Chez Jean (créé par les groupes Casino et Relay), alors que le modèle de l'hypermarché situé en périphérie des villes s'essouffle. Même les distributeurs peu présents jusqu'alors sur le créneau des supérettes se lancent dans la course avec des enseignes comme A 2 Pas (Auchan), U Express ou Intermarché Express. Le point de retournement peut être daté de 2008 avec le relèvement du seuil d'autorisation pour toute ouverture ou agrandissement de surfaces de vente de 300 à 1000m2 (Loi de Modernisation de l'Economie). Cela s'accompagne de la hausse du prix de l'énergie et de la prise de conscience progressive des citoyens des conséquences sur l'environnement de leurs comportements de consommation. Ces derniers se tournent désormais vers des magasins accessibles à pied offrant plus de praticité avec des produits du quotidien et des services de proximité plus nombreux (distribution servicielle), ce que les Anglo-saxons appellent "convenience".

Ces nouveaux concepts de centres villes, qu'ils relèvent de la domination par les coûts ou d'une stratégie de différenciation, ont construit leurs positionnements en exploitant différentes dimensions de la proximité qui vont au-delà de la simple distance géographique. Ils ont ainsi modernisé un concept, celui de la supérette, qui avait largement vieilli.

Cet article, de nature compréhensive, s'inscrit dans un volet de recherche sur la proximité, le commerce de détail alimentaire et sa logistique. Il fait suite à un premier travail qui fait le lien entre proximité et convenience en s'appuyant sur le cas des convenience stores japonais (Capo et Chanut, 2012). L'objectif est ici de proposer une grille de lecture analytique, fine et opérationnalisée, des sept dimensions de la proximité retenues pour analyser les positionnements voulus des nouveaux concepts de vente axés proximité ou convenience. Un 
focus particulier est fait sur la dimension inter-organisationnelle de la proximité qui permet de mettre l'accent sur l'organisation du canal de distribution (en amont comme en aval) et intègre les questions logistiques. Notre démarche est, après un retour sur les notions de positionnement voulu et de proximité, de présenter la grille d'analyse retenue, construite à partir d'une démarche abductive. La grille est ensuite utilisée pour analyser, à titre d'exemple, le positionnement voulu de trois enseignes (U express, Monoprix et Carrefour City Café), mais aussi pour identifier les points communs caractéristiques de la distribution de proximité moderne. La conclusion débouche sur l'idée que la grille d'analyse proposée peut-être utilisée au-delà des nouveaux concepts de proximité, pour analyser les positionnements d'autres formats, tels que l'hypermarché ou le drive.

\section{Différencier les positionnements voulus des commerces de proximité sous enseignes : proposition d'une grille de lecture de la proximité en 7 dimensions}

Face au développement des nouveaux concepts de proximité, l'observateur est tenté d'analyser le positionnement voulu par les groupes de distribution qui les ont lancés.

\section{Retour sur la notion de positionnement voulu}

Rappelons que le positionnement est la conception d'un produit/d'une concept d'enseigne, de son image dans le but de lui donner une place déterminée dans l'esprit des clients visés (Kotler et al., 2009). Il s'agit de conférer à la marque, ou marque-enseigne, une place singulière dans l'esprit du consommateur qui lui permet de se différencier de ses concurrents (Filser et al., 2012) et de délivrer une valeur accrue aux yeux du consommateur. Concernant le positionnement d'une enseigne de distribution, il est fréquent désormais de distinguer trois positionnements, respectivement celui qui est voulu par l'entreprise (ici le groupe de distribution qui lance le nouveau concept de proximité), celui qui est vécu par le personnel en 
contact et celui qui est perçu par le consommateur (Pontier, 1986 ; Dioux et Dupuis, 2009). Des distorsions entre ces trois positionnements sont en effet possibles, le positionnement voulu par l'enseigne n'étant pas toujours conforme à l'image que se fait effectivement le consommateur, ou le personnel en contact. Lazer et Kelley (1961) énoncent que le positionnement d'une enseigne s'exprime en termes de retailing-mix, avec trois composantes : le mix produits et services, le mix de la distribution physique -incluant les caractéristiques du point de vente- et le mix des communications-publicités, promotions et personnel en contact. Cependant, cette distinction est trop large et imprécise pour analyser en finesse les différences de positionnement des nouvelles enseignes de proximité. C'est pourquoi nous proposons une grille d'analyse de la proximité pouvant éclairer les choix de positionnements voulus observés dans le commerce, construite selon une démarche abductive.

\section{Méthodologie abductive et qualitative}

La grille de lecture du positionnement voulu, a été élaborée à partir d'une double source, théorique et empirique, exploitée de façon itérative. La revue de littérature, empruntant à des disciplines aussi diverses que l'économie, la psychologie sociale, la géographie, et les sciences de gestion, a permis de mettre en évidence le caractère multidimensionnel du concept de proximité, qui ne se limite pas aux aspects de distance géographique. Plusieurs dimensions ont été retenues et adaptées au contexte du commerce de détail. Par ailleurs, une analyse de contenu de la communication des groupes de distribution sur leurs enseignes de proximité a été menée. Divers documents constituant des sources d'évidence au sens de Yin (2003) ont été systématiquement analysés et codés : les rapports d'activités et de développement durable 2010 (RADD) des groupes de distribution lorsqu'ils existent (Carrefour, Casino, Monoprix, Auchan, Simply Market), à défaut les communiqués de presse et autres documents publiés à propos du lancement des nouveaux concepts (Intermarché, Leclerc, Système U) ; mais aussi les articles publiés dans la presse professionnelle de la distribution (LSA, Points de vente, 
Stratégies) et dans la presse généraliste (Le Monde, Les Echos, le Figaro). Ces sources reprennent en effet les discours des enseignes et sont donc fidèles aux positionnements voulus. L'analyse du contexte sémantique de la proximité (mots associés) dans les RADD et autres documents a permis d'opérationnaliser les différentes dimensions de la grille d'analyse de la proximité.

\section{Construction de la grille : depuis les variables théoriques de la proximité...}

La proximité est un construit multidimensionnel (Pecqueur et Zimmermann, 2004 ; Rychen et Zimmermann, 2008) qui relève de la subjectivité des acteurs (Le Blouch, 2001). Une distinction simple, reprise par Talbot et Kirat (2005), permet de présenter la proximité en deux volets singuliers (Torre et Rallet, 2005) : le volet spatial, dite aussi proximité géographique ou proximité d'accès, qui regroupe les proximités métrique, temporelle et circulatoire (Paché, 2006); et le volet non-spatial, traitant des proximités sociales, commerciales et institutionnelles, aussi appelé proximité organisée ou parfois proximité relationnelle (Rallet, 2002 ; Billaudot, 2004 ; Bouba-Olga et Grossetti 2008). En ce qui concerne l'analyse des commerces alimentaires et en sus du pan spatial de la proximité, Bergadaà et Del Bucchia (2009) ont décliné le pan non-spatial de la proximité en quatre dimensions qualifiées de proximité fonctionnelle, proximité relationnelle, proximité identitaire et proximité de processus. Ces quatre dimensions, centrées sur la seule perception du consommateur, dans un contexte helvétique, peuvent être complétées pour ajouter une autre dimension à la proximité non-spatiale : la proximité inter-organisationnelle, qui prend en compte l'organisation du canal de distribution en amont du consommateur (Capo et Chanut, 2012). Cette proximité inter-organisationnelle apparaît lorsque des liens étroits sont construits avec l'amont du canal pour concevoir ensemble les meilleurs produits du quotidien et une large palette de services facilitant la vie des habitants du quartier, et pour optimiser l'organisation de la logistique. En nous appuyant sur le modèle économique des convenience 
stores japonais, nous avons montré que la proximité inter-organisationnelle avec les acteurs de la supply chain participe à la construction d'un avantage concurrentiel distinctif autant du point de vue de l'organisation logistique que du marketing de l'enseigne et des produits. Les dimensions de la proximité identifiées dans la littérature ont été confrontées au discours des enseignes dans leurs communications et ont été complétées et opérationnalisées pour aboutir à une grille d'analyse fine de la proximité.

\section{...jusqu'à l'opérationnalisation des variables au sein de la grille d'analyse}

La grille d'analyse de la proximité (Tableau 1) issue de la démarche abductive reprend les 6 dimensions de la proximité -auxquelles nous ajoutons la proximité prix identifiée après analyse des sources d'évidence- leurs définitions et les variables retenues pour chaque dimension. Nous commentons ici les éléments les plus saillants de chaque dimension.

Concernant la proximité spatiale, nous ajoutons aux proximités métrique, temporelle et circulatoire, la distinction entre les concepts de proximité urbaine et rurale, car les groupes de distribution opèrent cette distinction. Concernant la proximité fonctionnelle, elle résulte à la fois de l'assortiment de produits et services et de toutes les facilités offertes (horaires étendus, livraison, drive) pour faire gagner du temps au consommateur et améliorer sa qualité de vie. Par exemple, la directrice d’Intermarché Express énonce que le concept a été pensé «autour de quatre attentes consommateurs : aller vite, trouver tout, avoir la vie facilitée et se sentir $b_{i e n^{2}} \gg$. Plusieurs distributeurs font un lien direct entre la proximité et l'adaptation de l'offre et insistent sur l'offre étendue de services. C'est le cas de Casino lorsqu'il souligne le «double sens de la proximité : géographique et en matière de services » (Casino, Rapport d'activité et de performances sociétales et environnementales 2010, p. 36).

\footnotetext{
${ }^{2}$ Article «Les Mousquetaires de la proximité », e-marketing.fr, Armelle Nebia, 7 janvier 2011, http://www.emarketing.fr/Article-A-La-Une/Les-Mousquetaires-de-la-proximite-2139.htm.
} 
La proximité relationnelle s'évalue à travers le nombre de salariés, leurs statuts, le niveau de conseil et d'attention porté aux clients. Certains distributeurs mettent en avant leur usage des nouveaux outils axés technologies de l'information et de la communication (TIC) pour renforcer le lien de proximité, à l'instar de SimplyMarket : «Les outils de dialogue vont se développer en 2011 par l'action de l'équipe web2.0 de SimplyMarket.[...il s'agit de] construire un site internet plus interactif pour créer avec les internautes le supermarché de demain et développer un blog par magasin afin de renforcer les liens de proximité » (SimplyMarket, Rapport développement durable 2010, p. 9). Cette utilisation participe aussi à la proximité fonctionnelle puisque les TIC permettent de consulter à distance et à n'importe quel moment des informations sur les points de vente (position géographique, offre...). Enfin, la proximité relationnelle est liée à la notion de confiance des acteurs. Ainsi, selon le directeur de la communication de Système U, les clients « ... savent que U est proche d'eux et ils font confiance aux offres et aux produits que l'on propose ».

La proximité identitaire se déploie à travers un design architectural distinctif mais surtout par la mise en avant de valeurs liées au développement durable (respect de l'environnement, approvisionnement local, responsabilité sociale des entreprises, etc.). Il convient en particulier de souligner le lien établi entre magasin de proximité et l'emploi local : «les magasins recrutent en effet près de $90 \%$ de leurs collaborateurs localement » (Carrefour, Rapport d'activité et de développement durable 2010, p. 50); le lien entre magasin de proximité et l'entreprenariat local : «Carrefour s'appuie également sur le développement de la franchise et notamment sur le dispositif de location-gérance en favorisant ainsi la création d'entreprises locales »; ou encore entre magasin de proximité et la solidarité : «Ce qui différencie SimplyMarket des autres distributeurs, ce sont ses valeurs de proximité et de solidarité, l'importance accordée aux hommes » (SimplyMarket, Rapport développement durable 2010, p. 12). 
Pour ce qui concerne la proximité de processus, deux éléments doivent être ajoutés à l'axe contrôle/traçabilité et à l'axe fraîcheur : le poids des marques de distributeurs (MDD) dans l'assortiment, présenté comme un moyen à la fois de contrôler la qualité et d'être proche des clients : «[pour] l'enseigne de proximité A 2 Pas [...] centrée sur les produits frais et la marque Auchan, son offre s'adapte aux particularités des quartiers » (Auchan, Rapport d'activités 2011, p. 16) ; la mise en avant par certains distributeurs de la faible distance parcourue par les produits entre les lieux de production et les lieux de distribution : «chez $U$, pour réduire le prix, on a aussi réduit les distances. Oui, en faisant élaborer $82 \%$ de nos produits alimentaires près de chez vous, par des entreprises françaises » (Publicité télévisuelle de Système $U$ ). Cet élément est d'ailleurs souvent associé à la proximité interorganisationnelle et à la volonté de privilégier des partenariats en amont avec des producteurs locaux ou façonniers de MDD, et en aval avec des distributeurs associés ou franchisés : « $L a$ densité extrême du réseau de points de vente des Mousquetaires donne au Groupement un ancrage local particulièrement fort. Tout comme les responsables de PME, attachés à leur terroir et à leur région. Ces valeurs partagées viennent renforcer cette proximité naturelle, faite du même désir d'entreprendre, du même souci d'indépendance et de cette volonté commune de relever les mêmes défis économiques et sociaux. Les Mousquetaires demeurent persuadés du bien-fondé $d u$ "réussir ensemble ». (Intermarché, dossier de presse «Charte Fournisseurs », septembre 2010).

Enfin, aux six dimensions liées à la proximité, nous ajoutons le positionnement prix, qui reste une variable clé de différenciation des concepts de commerce de détail. Le lien avec la proximité est assez direct car le choix d'une politique de prix adéquate et un bon rapport qualité-prix au regard de l'offre proposée participent au sentiment de proximité vis-à-vis de l'enseigne, ce qui permet de parler de «proximité prix ». Classiquement, deux stratégies de 
prix sont observées : celle des prix bas toute l'année (Every day low price ou EDLP) et celle d'un niveau de prix plus élevé avec promotions ponctuelles (High-Low).

\section{Tableau 1 - Grille d'analyse des 7 dimensions de la proximité : définitions et variables [Insérer ici le Tableau 1]}

Au total, sept dimensions de la proximité sont mobilisées pour analyser le positionnement des enseignes. Deux remarques doivent accompagner la présentation de cette grille d'analyse. D’une part, si nous avons classé le poids des MDD dans la dimension «processus » de la proximité, en réalité l'offre de MDD, centrale dans ce format, permet aux enseignes de jouer sur plusieurs dimensions de la proximité : les proximités fonctionnelle, de processus, interorganisationnelle, identitaire et prix. En effet, les MDD donnent un choix large d'approvisionnement et distinctifs des autres enseignes pour les commerçants [proximité

fonctionnelle], tout en permettant un positionnement «bon rapport qualité prix » [proximité prix]. Les MDD portent souvent le nom de l'enseigne et sont ainsi garantes du contrôle de la qualité des produits par le distributeur [proximité de processus], et de sa capacité à adapter son offre aux besoins spécifiques de la clientèle et des zones de chalandises des points de vente. Par ailleurs, leur conception et fabrication étant réalisées à partir d'un cahier des charges «négocié » avec les fournisseurs façonniers de MDD (le plus souvent des PME), les MDD constituent un vecteur d'échange de connaissances et d'informations entre les membres de la supply chain sur les besoins réels et émergents des consommateurs [proximité interorganisationnelle]. Enfin, si les distributeurs offrent une large gamme de MDD, des MDD Discount au MDD premium, certaines s'ancrent dans une logique identitaire en développant des thématiques : éthiques, locales, régionales, bio... ou en étant le support d'actions tournées 
vers le développement durable (étiquetage de l'empreinte carbone sur les MDD dans le groupe Casino par exemple) [proximité identitaire].

D'autre part, dans le discours des distributeurs, sont associées souvent plusieurs dimensions de la proximité. Ainsi, le texte suivant issu du RADD 2010 de Carrefour (p. 49), renvoie à 3 dimensions de la proximité : «Carrefour teste en 2010 l'achat direct de produits par ses magasins auprès de producteurs locaux [proximité inter-organisationnelle amont]. Elle vise à développer une offre de "paniers de saison » [proximité relationnelle : adaptation de l'offre], de marques «petits producteurs»[proximité inter-organisationnelle amont], et répondre ainsi aux attentes des consommateurs soucieux d'accéder à des produits de qualité [proximité de processus]. Dans la partie suivante, nous utilisons la grille de lecture proposée pour analyser le positionnement voulu de trois enseignes de proximité.

\section{Points communs et spécificités des positionnements voulus des concepts de proximité sous enseignes}

Les nouveaux concepts de la proximité lancés récemment par les grands distributeurs français présentent un certain nombre de points communs, les rapprochant des convenience stores à la japonaise. Ils sont principalement situés dans les zones urbaines, en centres villes, donc proches des domiciles ou des bureaux [proximité spatiale : métrique et temporelle]. Ils offrent une large gamme de produits prêts à consommer et de produits frais préemballés, des zones snacking ou autres espaces mange debout, leurs horaires d'ouverture s'étendent, leurs offres de services s'étoffent. De surfaces limitées (100 à $600 \mathrm{~m} 2)$, ils permettent des achats rapides et efficaces [proximité fonctionnelle]. Au-delà, ils reposent sur trois piliers centraux et communs aux différentes enseignes, que nous exposons rapidement avant d'analyser les positionnements voulus de trois enseignes : U express, Monoprix et Carrefour City Café. 
Les trois piliers centraux communs aux différentes enseignes de proximité : une structure de coûts et logistique compliquées, la franchise, les MDD

Les nouveaux concepts de proximité ont une structure de coûts élevés et des contraintes logistiques fortes. Ils ont fait le choix de la franchise et d'un assortiment centré sur les MDD.

Une structure de coûts et logistique compliquées. Trois contraintes fortes pèsent sur la rentabilité et rend la proximité «compliquée », pouvant être résumées par les «trois L »: une Localisation en centres-villes, avec des Loyers élevés (pouvant atteindre 6 ou $7 \%$ du chiffre d'affaires) et une Logistique compliquée du fait du manque de fluidité de la circulation et de l'encombrement des voieries en centre-ville, sans compter les nuisances en termes de pollution induite par les activités logistiques et un déficit certain des aires de livraisons en rapport avec les besoins. Certains distributeurs innovent en matière de logistique urbaine, notamment en adoptant des solutions de transport multimodal, à l'instar de Monoprix ou de Franprix (groupe Casino) qui combinent transport par voies fluviale et ferroviaire pour l'entrée des marchandises au cour de Paris et par camions (au gaz pour Monoprix) pour le dernier kilomètre (Chanut et Paché, 2013). Ou encore en commençant à initier des solutions de mutualisation horizontale (entre distributeurs, éventuellement concurrents) des ressources logistiques, pour entrer dans la ville. Le mouvement reste toutefois timide, les grands distributeurs alimentaires français ayant construit des Supply Chain distinctes, avec le souci premier de dégager un avantage concurrentiel par rapports aux chaînes logistiques concurrentes. Il en résulte des freins pour coopérer. Cela devra toutefois évoluer, si l'objectif est l'économie de ressources (écologiques et économiques). Les frais logistiques des magasins de proximité représentent déjà jusqu'à $12 \%$ du chiffre d'affaires, contre $8 \%$ pour un super et $4 \%$ pour un hyper (LSA n ${ }^{\circ} 2167$, février 2011). Et ils ne peuvent qu'augmenter, d'autant que l'offre de ces magasins comprend une partie importante de produits frais, de produits snacking à date limite de consommation (DLC) courtes et de fruits et légumes exigeants à gérer au plan logistique. Au total, le niveau de charges des enseignes de proximité serait de 10 
à $30 \%$ plus élevé qu'en supermarché, et il ne peut pas toujours être compensé par un niveau de prix de vente plus élevé.

La franchise, soutien d'un modèle de développement. Force est de constater que les groupes de distribution français s'appuient largement sur les commerçants indépendants pour développer leurs points de vente en proximité. C'est le cas des groupes du commerce associé (Système U, Leclerc, Intermarché) qui culturellement se développent avec des commerçants indépendants au plan juridique et financier. Cependant, la forme organisationnelle franchise est aussi choisie pour le format proximité par des groupes de distribution de culture plutôt succursaliste. C'est le cas des acteurs historiques de la proximité tels que Casino ou Carrefour qui développent leurs concepts de proximité majoritairement en franchise alors que leurs autres formats de magasins (hypermarchés, supermarchés, hard discount) sont intégrés ou mixtes. Plus remarquable encore est le cas de groupes de distribution à forte culture succursaliste, tel Monoprix, qui ouvre ses Dailymonop' et Monop' en franchise. Cela lui permet une croissance plus rapide du réseau qu'un développement en succursales (Boulay et Chanut, 2010).

Des MDD au cœur de l'offre de proximité. L'offre de produits de chaque enseigne de proximité s'appuie fortement sur la composante MDD. Ainsi, si le groupe Carrefour affiche $40 \%$ de MDD pour ses formats de proximité, U Express ou Casino annoncent $50 \%$ et A 2 Pas 70\%. Nous avons déjà souligné la puissance des MDD pour asseoir le positionnement voulu d'une enseigne en termes de proximités fonctionnelle, de processus, inter-organisationnelle, identitaire et prix.

Analyse des positionnements voulus de trois enseignes de proximité à l'aide de la grille de lecture proposée

Au-delà de ces points communs, les enseignes de proximité cherchent à se différencier sur différentes dimensions de la proximité. Nous l'illustrons avec l'analyse du positionnement de 
trois cas : U Express, Monoprix et Carrefour City Café. Pour chaque enseigne, nous rappelons le contexte (éléments sur le groupe de distribution, son organisation, sa stratégie en matière de proximité), avant de présenter le positionnement à l'aide de la grille d'analyse des dimensions de la proximité. Sur chaque dimension, un degré de proximité (faible, moyen, élevé) a été attribué par deux chercheurs, séparément puis en confrontant leurs analyses. Il a été apprécié de manière qualitative et relative à partir du discours comparé des enseignes. Les schémas en « radar » ou « spider web » visuellement très parlants illustrent le positionnement voulu et permettent une comparaison visuelle des différents profils de proximité des enseignes.

\section{U Express de Système U-Ancrage local et solidaire}

Quatrième groupe de distribution alimentaire en France avec environ 1400 points de vente, Système U possède une palette de formats de magasins allant de l'hypermarché aux magasins de proximité. Le réseau se déploie à travers 613 points de vente de proximité, dont 95 magasins sous l'enseigne Marché U (en voie de modernisation), 368 magasins sous l'enseigne Utile et 150 magasins sous une nouvelle enseigne U Express en forte croissance. Système U est une structure coopérative regroupant de nombreux magasins indépendants où les associés, propriétaires de leurs fonds, possèdent chacun une part au moins dans la structure tête de réseau. Système U organise ses achats autour de quatre grandes centrales d'achat régionales et a développé des partenariats avec des coopératives agricoles (comme Biolait, par exemple). La proximité est au cœur de la philosophie et de l'organisation de Système U avec une emphase sur les notions de «local » et de «solidarité » qui forge sa proximité identitaire. Ces valeurs lui confèrent une identité distinctive forte, qui s'illustrent à quatre niveaux : au niveau de la proximité inter-organisationnelle aval avec les patrons de magasins associés, au niveau de la proximité inter-organisationnelle amont, à travers le partenariat avec des fournisseurs locaux, et au niveau de la proximité de processus, à travers un assortiment largement axé sur 
des produits locaux, sains, maîtrisés, en grande partie des MDD, avec un positionnement prix discount qui renforce la proximité prix. Ces valeurs de Système U se retrouvent dans les choix de positionnement distinctif d'U Express, et son nouveau concept décrit dans le tableau 2.

Tableau 2 - Analyse des dimensions de la proximité de U Express

[Insérer ici le tableau 2]

Les autres dimensions de la proximité sont moins saillantes chez U Express : la proximité spatiale se décline plus par une facilité d'accès que par une présence en centre-ville. Au niveau fonctionnel, une offre large de références et services n'est cependant pas soutenue par des horaires d'ouverture très larges. La proximité relationnelle, mobilisée à travers la notion de confiance, n'est pas prépondérante.

Figure 1 : Profil de la proximité de U Express

[Insérer ici la figure 1]

\section{Monoprix - Relationnel et développement durable}

Acteur historique de la proximité présent seulement au centre-ville des grandes et moyennes villes françaises, Monoprix appartient pour moitié au groupe Casino et aux Galeries Lafayette $^{3}$. L'enseigne possédait en 2011, 514 points de vente dont 295 Monoprix (magasins historiques, intégrés), mais a lancé récemment de nombreux nouveaux concepts de proximité. Elle compte, en 2011, 51 Monop', 13 Dailymonop', 49 Naturalia (spécialisés dans le bio) et quelques Monop'Station en partenariat avec Servex dans 3 gares françaises (Strasbourg, Chartres et Thionville). Un tiers seulement des points de vente du groupe sont des franchises

\footnotetext{
${ }^{3}$ Le rachat total annoncé du capital de Monoprix, par l'acquisition de la participation de $50 \%$ des Galeries Lafayette en 2013, devrait renforcer encore le leadership du groupe Casino en matière de proximité.
} 
en 2011 mais Monoprix affiche son souhait de se développer en franchise, pour les nouveaux concepts de petites surfaces. L'enseigne développe l'idée d'avoir un maximum de biens et de services «sous le même toit» et se déclare leader de la proximité. Son positionnement distinctif repose sur une proximité spatiale (emplacements en hyper centre-ville, fruit de son histoire, même si l'accès voiture est peu aisé), une proximité fonctionnelle (offre large, de qualité, avec des prix plutôt élevés, avec un développement notable de services de confort, une amplitude horaire étendue), mais aussi les proximités relationnelle et identitaire illustrée par l'expression «proximité de cour» (RADD, 2010). Monoprix insiste particulièrement sur les sentiments d'appartenance et de confiance.

Tableau 3 - Analyse des dimensions de la proximité de Monoprix

[Insérer ici le tableau 3]

La proximité relationnelle, force historique de l'enseigne, s'appuie sur un personnel en contact plus nombreux, tissant des liens forts avec la clientèle et un service client soucieux de créer des services et du lien permanent avec le cœur de cible, y compris en utilisant les dernières TIC (application Smartphone). La proximité identitaire, autre fer de lance du concept, se traduit par une offre et un discours tournés vers le développement durable (MDD de produits bio, équitables notamment), mais aussi par des solutions innovantes en matière de logistique urbaine. Monoprix a ainsi été la première enseigne de distribution alimentaire à développer, dès 2007, avec sa filiale logistique Samada, une solution de transport multimodal pour limiter les nuisances dues à l'entrée des marchandises dans Paris. L'approvisionnement des magasins au cœur de Paris se fait par voie fluviale jusqu'au Havre, puis par train sur 30 kilomètres entre Le Havre et le port de Bercy, et enfin, par camions au gaz pour le dernier kilomètre. Si cette solution a entraîné un surcoût logistique par rapport au transport tout 
camion estimé par l'enseigne à 15-20\% en raison des ruptures de charge qu'elle induit, elle permet aussi d'afficher 12000 camions de moins dans Paris, et une économie de 337 tonnes de dioxyde de carbone $\left(\mathrm{CO}_{2}\right)$ et de 19 tonnes d'oxyde d'azote $\left(\mathrm{NO}_{\mathrm{x}}\right)$ par an (RADD Monoprix, 2008, 2011). Elle renforce donc la proximité identitaire «développement durable » de Monoprix.

Figure 2 : Profil de la proximité de Monoprix

[Insérer ici la figure 2]

\section{Carrefour City Café - la convenience, avec snacking et dépannage}

Acteur historique de la proximité, le groupe Carrefour génère $10 \%$ de son CA dans ses points de vente de proximité en 2010. En 2011, sur un parc total de 4631 points de vente, la proximité représente 3285 magasins. Le groupe a adopté récemment une «stratégie multiformats mono-marque» en déclinant la marque Carrefour pour tous ses formats. Le changement de noms d'enseignes a été l'occasion de revisiter les concepts de vente dits de proximité, pour les rendre plus attractifs, rajeunir leurs images, et ainsi générer de la croissance pour compenser l'essoufflement des hypermarchés. De nouveaux concepts ont ainsi été créés sous les enseignes Carrefour City, Carrefour Express, Carrefour Contact, amenés à remplacer en partie les anciens concepts de supérettes Shoppi, Huit à Huit et Marché plus. Parallèlement, le groupe a décidé de commercialiser des MDD à la marque Carrefour dans tous ses formats. Carrefour a aussi lancé, en test, un nouveau concept sous l'enseigne Carrefour City Café. Si le test a échoué à Bordeaux, un point de vente est encore exploité à Paris (Jussieu). 
Le positionnement de proximité de Carrefour City Café ne cherche pas à exploiter de façon maximale toutes les dimensions de la proximité mais propose un concept de spécialité s'appuyant sur trois dimensions principales: les proximités spatiale, fonctionnelle et de processus.

Tableau 4 - Analyse des dimensions de la proximité de Carrefour City Café [Insérer ici le tableau 4]

Il est tourné vers le snacking et le dépannage (à l'instar des convenience stores à la japonaise) situés en hyper-centre ou dans des zones à fort trafic, ouverts 7 jours sur 7 jusqu'à tard dans la nuit, et proposant des produits prêts à consommer ultra-frais, avec peu de références (essentiellement des MDD) ainsi que des espaces pour consommer sur place.

Figure 3 : Profil de la proximité de Carrefour City Café

[Insérer ici la figure 3]

Ainsi, les nouveaux formats de proximité reposent sur des piliers -logistique et structure de coûts, MDD et franchise- qui forment l'ossature du modèle économique. Cette ossature se distingue des autres formats de distribution et représente une première strate de différenciation. Au-delà, les distributeurs cherchent à développer des positionnements différenciables les uns des autres par le consommateur au sein même des enseignes de proximité et de proposer des combinaisons différentes des dimensions de la proximité comme cela a été montré au travers des trois cas analysés. Cette deuxième strate de différenciation permet aux groupes de la distribution de construire des profils variables de proximité, qu'ils jouent sur un grand nombre de dimensions (Monoprix et U Express) ou à l'extrême inverse sur quelques dimensions choisies (Carrefour city Café).

Figure 4 : Axe de différenciation des concepts de distribution par la proximité 
[Insérer ici la figure 4]

\section{Conclusion}

Cet article avait pour objectif d'analyser le positionnement des nouveaux concepts de commerce de proximité alimentaires qui fleurissent actuellement en France sous différentes enseignes. Il propose une grille de lecture de la proximité, intégrant, grâce à une démarche abductive (revue de littérature multidisciplinaire, analyse du contenu des communications des enseignes) sept dimensions riches : le spatial, le fonctionnel, le relationnel, l'identitaire, le processus, l'inter-organisationnel et le prix. La grille de lecture a ensuite été mobilisée pour analyser, à titre d'illustration, le positionnement distinctif de trois enseignes : U Express, Monoprix, et Carrefour City Café.

Toutefois, au-delà des concepts dits de proximité, la grille élaborée pourrait être mobilisée pour analyser et comparer les positionnements voulus d'autres enseignes ayant adopté d'autres formats de distribution (le supermarché, le hard discount par exemple); ou pour comprendre le déclin de certains d'entre eux (l'hypermarché); ou encore, pour expliquer l'essor de nouvelles formes de distribution comme le drive. En effet, ce nouveau canal de distribution, qui emprunte à la fois au canal physique (livraison dans un lieu physique) et au canal virtuel (commande par internet), investit pleinement les dimensions spatiales et fonctionnelles de la proximité : implantés sur des lieux de passage commodes (proximité d'accès), ils proposent de faciliter la vie du consommateur par du service : la préparation de sa commande ce qui suppose l'internalisation des activités de manutention (jusque là opérée par le chaland lorsqu'il fait ses courses) (Rouquet et al., 2010) et une amplitude horaire large pour le retrait des marchandises. La proximité inter-organisationnelle est aussi questionnée car le drive suppose de revisiter les relations avec les acteurs de la filière et d'adapter l'organisation logistique en y intégrant le consommateur. De plus, un prolongement et complément de cette 
analyse de nature compréhensive pourrait consister à comparer le positionnement voulu par les enseignes à celui vécu par le personnel en contact ainsi que par celui perçu par la clientèle. La grille proposée est donc de portée plus importante que l'analyse des positionnements des nouveaux concepts de proximité lancés pour rénover les superettes. D'autant que le concept de proximité semble central dans la compréhension des exigences nouvelles du consommateur du début du XXIème siècle (Moati et al., 2010). Les distributeurs l'ont bien compris et mobilisent le concept de proximité au delà du format du même nom. Ils devront toutefois être attentifs à maintenir une certaine différenciation entre leurs formats de commerce, au risque de lasser le consommateur. En effet, les stratégies mono-marque, voire mono-enseigne, de certains distributeurs comme Carrefour ou Casino, ainsi que la présence accrue des MDD communes vendues dans tous les formats de l'enseigne, tendent à une homogénéisation plutôt qu'à une différenciation tant dans le discours des distributeurs que dans l'offre proposée. Or une trop grande homogénéisation peut entraîner des effets négatifs dans la perception des consommateurs notamment s'ils considèrent qu'il ne leur est plus offert assez de diversité. La réflexion et les tests actuels du groupe Carrefour, quelques années à peine après l'adoption de la convergence des marques-enseignes, illustrent ce risque de manque de diversité (LSA, $\left.\mathrm{n}^{\circ} 2240,2012\right)$.

\section{Références}

Bergadaà, M., Del Bucchia, C., (2009), La recherche de proximité par le client dans le secteur de la grande consommation alimentaire, Management \& Avenir, n²1, pp. 121-135.

Billaudot, B., (2004), Proximité, réseaux et coordination industrielle : quelle coordination conceptuelle?, Quatrièmes Journées de la proximité, Marseille (France), 17-18 juin.

Bouba-Olga, O., Grossetti, M., (2008), Socio-économie de proximité, Revue d'Economie Régionale et Urbaine, $\mathrm{n}^{\circ} 3$, pp. 311-328.

Boulay, J., Chanut, O., (2010), Les réseaux de franchise, La Découverte, Collection Repères. 
Capo, C., Chanut, O., (2012), Quand la proximité crée la convenience : une grille de lecture du système de distribution japonais, Les Cahiers Scientifique du Transport, n ${ }^{\circ}$ 61, pp. 91-117.

Chanut, O., Paché, G., (2013), Le PSL à l'épreuve de la logistique urbaine : des signaux faibles porteurs de profondes mutations, Logistique \& Management, Vol. 21, n 1, pp. 31-44.

Dioux, J., Dupuis, M., (2009), La distribution - Stratégies des groupes et marketing des enseignes, Pearson Education.

Filser, M., Des Garets, V., Paché, G., (2012), La distribution : organisation et stratégie, Editions Management \& Société, Caen, 2e éd.

Kotler, P., Keller, K., Brady, M., Goodman, M., Hansen, T., (2009), Marketing Management, Pearson Education.

Lazer, W., Kelley, E., (1961), The Retailing Mix: Planning and Management, Journal of Retailing, pp. 34-41

Le Blouch, G., (2001), Approche systémique de la proximité : définition et discussion, Troisièmes journées de la proximité, Paris (France), 13-14 décembre.Moati, P., Jauneau, P., Lourdel, V., (2010), Quel commerce pour demain ? Cahier de Recherche, n²71, Centre de Recherche pour l'Etude et l'Observation des Conditions de Vie (CREDOC), Paris, novembre.

Paché, G., (2006), Approche spatialisée des chaînes logistiques étendues- De quelle(s) proximité(s) parte-t-on ?, Les Cahiers Scientifiques du Transport, $\mathrm{n}^{\circ} 49$, pp. 9-28.

Pecqueur, B., Zimmermann, J. B., (2004), Economie de Proximités, Lavoisier.

Pontier, S., (1986), Le positionnement vécu : une variable clef du positionnement du point de vente, 2ème congrès de l'Association Française de Marketing, 29-30 janvier.

Rallet, A., (2002), L'économie des proximités. Propos d'étape, Etudes et Recherches sur les Systèmes Agraires et le Développement., Vol. 33, pp. 11-25.

Rouquet, A., Goudarzi, K., Henriquez, T., (2010), Le transfert d'activités logistiques entreprise-consommateur : une comparaison des cas Auchan Drive et IKEA, Logistique \& Management, Vol. $18 \mathrm{n}^{\circ} 2$, pp. 13-25.

Rychen, F., Zimmermann, J.-B., (2008), Clusters in the Global Knowledge-based Economy: Knowledge Gatekeepers and Temporary Proximity, Regional Studies, Vol. 42, nº, pp. 767776.

Talbot, D., Kirat, T., (2005), Proximités et institutions: nouveaux éclairages, Economie et Institutions, $\mathrm{n}^{\circ} 6$ and $7,1_{\text {st }}$ and 2 nd half-years.

Torre, A., Rallet, A., (2005), Proximity and Localization, Regional Studies, Vol. 39, n¹, pp. 47-60.

Yin, R.K., (2003), Case Study Research, Design and Methods, Sage. 


\section{Tableau 1}

\begin{tabular}{|c|c|c|}
\hline $\begin{array}{l}\text { DIMENSIONS } \\
\text { DE LA } \\
\text { PROXIMITE }\end{array}$ & $\begin{array}{l}\text { DEFINITIONS DES } \\
\text { DIMENSIONS }\end{array}$ & $\begin{array}{l}\text { VARIABLES RETENUES POUR ANALYSER LE } \\
\text { POSITIONNEMENT VOULU }\end{array}$ \\
\hline $\begin{array}{l}\text { PROXIMITE } \\
\text { SPATIALE OU } \\
\text { D'ACCES }\end{array}$ & $\begin{array}{l}\text { Faible distance physique ou } \\
\text { temps d'accès, facilité d'accès. } \\
\text { La Proximité spatiale est } \\
\text { perçue différemment entre ville } \\
\text { et en milieu rural. }\end{array}$ & $\begin{array}{l}\text {-Métrique : implantation proche des lieux de vie (domicile, bureau) } \\
\text { versus périphérie } \\
\text {-Temporelle : implantation dans lieux à fort trafic/passage (gare, } \\
\text { métro) } \\
\text { •Circulatoire : facile d'accès, parking aisé } \\
\text {-Mais aussi distinction : rural versus urbain }\end{array}$ \\
\hline $\begin{array}{c}\text { PROXIMITE } \\
\text { FONCTIONNELLE }\end{array}$ & $\begin{array}{l}\text { Deux aspects dans } \\
\text { l'appréciation de la proximité } \\
\text { fonctionnelle : } \\
\text { - l'« offre » des } \\
\text { produits/services disponibles } \\
\text { et adaptés aux besoins de la } \\
\text { zone de chalandise } \\
\text { - la «commodité » avec des } \\
\text { horaires d'ouverture larges. }\end{array}$ & $\begin{array}{l}\text {-Profondeur de l'offre : offre large (plusieurs familles de produits) } \\
\text { versus étroite (alimentaire principalement) } \\
\text { - Offre snacking et produits à consommer préemballés : large versus } \\
\text { étroite } \\
\text { - Offre de produits ultrafrais (viande, fruits et légumes) : large versus } \\
\quad \text { étroite } \\
\text {-Quantité de services offerts : peu de services versus beaucoup de } \\
\text { services annexes } \\
\text {-Etendue des horaires d'ouverture : } 9 \mathrm{~h}-19 \mathrm{~h} \text { versus des horaires plus } \\
\text { étendus }\end{array}$ \\
\hline $\begin{array}{c}\text { PROXIMITE } \\
\text { RELATIONNELLE }\end{array}$ & $\begin{array}{l}\text { Développement de sentiments } \\
\text { de confiance et d'attachement } \\
\text { entre l'enseigne et le } \\
\text { consommateur, via le } \\
\text { personnel en contact et/ou } \\
\text { l'utilisation des TIC } \\
\text { relationnels. }\end{array}$ & $\begin{array}{l}\text {-Importance du personnel en contact (nombre, CDI ou CDD, temps } \\
\text { plein ou partiels) } \\
\text {-Mise en avant du conseil, du respect des clients, écoute attentive, } \\
\text { adaptation à la demande } \\
\text {-Mise en avant de l'usage d'outils modernes (Internet, smartphones) } \\
\text { pour entretenir liens et contacts (réseaux sociaux, } \\
\text { m-commerce,etc.) }\end{array}$ \\
\hline $\begin{array}{l}\text { PROXIMITE } \\
\text { IDENTITAIRE }\end{array}$ & $\begin{array}{l}\text { Développement d'un } \\
\text { sentiment d'appartenance à } \\
\text { une communauté, né d'un lien } \\
\text { affectif, conatif et cognitif. } \\
\text { Partage de valeurs autour du } \\
\text { bio, de l'éthique, du local } \\
\text { notamment. }\end{array}$ & $\begin{array}{l}\text {-Mise en avant d'actions spécifiques originales « environnement »: } \\
\text { marquage } \mathrm{CO} 2 \text { des produits } \\
\text {-Mise en avant d'actions spécifiques originales « éthique » : label } \\
\text { commerce équitable } \\
\text { •Mise en avant de produits bios en plus grande quantité } \\
\text {-Mise en avant respect des salariés, insertion professionnelle des } \\
\text { personnes handicapées } \\
\text {-Mise en avant d'organisation logistique optimum (moins de } \\
\text { nuisances) } \\
\text {-Identité forte : design architectural original, marqué, spécifique }\end{array}$ \\
\hline $\begin{array}{l}\text { PROXIMITE DE } \\
\text { PROCESSUS }\end{array}$ & $\begin{array}{l}\text { Mise en place d'une } \\
\text { traçabilité et d'un processus } \\
\text { de production garantissant la } \\
\text { qualité de l'offre. Le poids des } \\
\text { MDD et la faible distance } \\
\text { entre lieux de production et } \\
\text { lieux de distribution. }\end{array}$ & $\begin{array}{l}\text {-Mise en avant de la traçabilité et des contrôles qualité } \\
\text { - Mise en avant de la fraîcheur des produits (produits frais et prêts à } \\
\text { la consommation à dates de péremption très courtes) } \\
\text {-Mise en avant de la faible distance entre lieux de production et de } \\
\text { distribution } \\
\text {-Poids des MDD dans l'assortiment (MDD, caution de l'enseigne } \\
\text { qui engage sa réputation) }\end{array}$ \\
\hline $\begin{array}{c}\text { PROXIMITE } \\
\text { INIER- } \\
\text { ORGANISATIONNEULE }\end{array}$ & $\begin{array}{l}\text { Mise en place de partenariats } \\
\text { entre distributeurs et les } \\
\text { acteurs en amont et aval de la } \\
\text { Supply Chain afin de mieux } \\
\text { maîtriser assortiments et } \\
\text { logistique. }\end{array}$ & $\begin{array}{l}\text {-Mise en avant de partenariats avec producteurs locaux (proximité } \\
\text { inter-organisationnelle amont) } \\
\text { •Mise en avant de partenariats avec façonniers de MDD, industriels } \\
\text { (proximité inter-organisationnelle amont) } \\
\text { •Mise en avant de partenariats en aval (distributeurs franchisés ou } \\
\text { associés) (proximité inter-organisationnelle aval) }\end{array}$ \\
\hline $\begin{array}{l}\text { PROXIMITE } \\
\text { PRIX }\end{array}$ & $\begin{array}{l}\text { Choix d'une politique de prix } \\
\text { adéquate. Un bon rapport } \\
\text { qualité-prix au regard de } \\
\text { l'offre proposée. }\end{array}$ & $\begin{array}{l}\text {-Mise en avant du positionnement prix discount versus } \\
\text { positionnement prix élevé } \\
\text {-EDLP (Every Day Low Price) versus high-Low }\end{array}$ \\
\hline
\end{tabular}




\section{Tableau 2}

\begin{tabular}{|c|c|c|}
\hline $\begin{array}{l}\text { DIMENSIONS DE LA } \\
\text { PROXIMITE }\end{array}$ & DESCRIPTIF D'U EXPRESS & $\begin{array}{l}\text { DEGRE DE } \\
\text { PROXIMITE } \\
\text { DANS LE } \\
\text { DISCOURS }\end{array}$ \\
\hline $\begin{array}{l}\text { PROXIMITE SPATIALE } \\
\text { OU D'ACCES }\end{array}$ & $\begin{array}{l}\text {-Implantations rurales et urbaines en périphérie des centres-villes } \\
\text { (donc peu en hyper centre-ville). } \\
\text {-Facilité d'accès par les transports en commun et la voiture : } \\
\text { proximité circulatoire et temporelle. }\end{array}$ & Moyen \\
\hline $\begin{array}{c}\text { PROXIMITE } \\
\text { FONCTIONNELLE }\end{array}$ & $\begin{array}{l}\text {-Offre large de produits frais (6000 à } 8000 \text { références). } \\
\text { •De nombreux services. }\end{array}$ & Moyen \\
\hline $\begin{array}{c}\text { PROXIMITE } \\
\text { RELATIONNELLE }\end{array}$ & $\begin{array}{l}\text {-Discours axé sur la confiance entre les clients et les } \\
\text { commerçants, renforcée par la forme organisationnelle. }\end{array}$ & Moyen \\
\hline $\begin{array}{l}\text { Proximite } \\
\text { IDENTITAIRE }\end{array}$ & $\begin{array}{l}\text {-Identité forte construite autour de l'axe du développement local } \\
\text { et solidaire : production locale, insertion dans le tissu industriel et } \\
\text { commercial local, produits sains et maîtrisés. }\end{array}$ & Elevé \\
\hline $\begin{array}{l}\text { Proximite de } \\
\text { PROCESSUS }\end{array}$ & $\begin{array}{l}\text { •Partenariats et nombreuses MDD (U Saveurs, « Made in } \\
\text { France », bio...) présentés comme garantie de qualité et } \\
\text { traçabilité des produits et «créateurs d'identité ». } \\
\text {-Le groupement est en train de repenser totalement la } \\
\text { composition de ses produits U, afin de les rendre plus sains, en } \\
\text { passant notamment au crible } 80 \text { des composants (Parabène, } \\
\text { bisphénol, aspartam, huile de palme, sel d'aluminium...) dont } \\
\text { l'usage est actuellement en débat. L'objectif est de faire de sa } \\
\text { marque propre des produits de préférence au même titre que } \\
\text { certaines grandes marques nationales. }\end{array}$ & Elevé \\
\hline $\begin{array}{l}\text { PROXIMITE INTER- } \\
\text { ORGANISATIONNELLE }\end{array}$ & $\begin{array}{l}\text { •Des associés indépendants, stables et implantés qui peuvent } \\
\text { tisser des liens durables avec les clients. } \\
\text { •Echanges importants entre les associés, favorisés par des } \\
\text { réunions mensuelles et un institut de formation interne (Force U) } \\
\text { dans le but affiché de créer une réelle vie associative. De plus, } \\
\text { des tandems sont formés entre un nouvel adhérent et un } \\
\text { permanent (parrainage). } \\
\text { •Choix de travailler avec des fournisseurs locaux. En } 2009 \text { et } \\
\text { 2010, plusieurs structures dédiées à la mise en place de } \\
\text { partenariats avec les producteurs locaux se sont développées au } \\
\text { sein des régions Alsace, Sud-Ouest, ou Vendée... } \\
\text {-Plateformes logistiques régionales. } \\
\text {-MDD développées conjointement avec les façonniers, pour le } \\
\text { bio, le « Made in France », le régional... }\end{array}$ & $\begin{array}{l}\text { Elevé } \\
\text { en amont et } \\
\text { en aval }\end{array}$ \\
\hline PROXIMITE PRIX & $\begin{array}{l}\text { - EDLP : prix bas toute l'année. } \\
\text { - Lien établi entre production locale, faibles distances et prix bas. }\end{array}$ & Elevé \\
\hline
\end{tabular}


Tableau 3

\begin{tabular}{|c|c|c|}
\hline $\begin{array}{l}\text { DIMENSIONS DE LA } \\
\text { PROXIMITE }\end{array}$ & DESCRIPTIF DE MONOPRIX & $\begin{array}{l}\text { DEGRE DE } \\
\text { PROXIMITE } \\
\text { DANS LE } \\
\text { DISCOURS }\end{array}$ \\
\hline $\begin{array}{l}\text { Proximite SPATIALE } \\
\text { OU D'ACCES }\end{array}$ & $\begin{array}{l}\text { Proximité spatiale élevée acquise pour le groupe du fait de sa } \\
\text { présence historique dans les centres-villes, même si problème de } \\
\text { parking parfois. }\end{array}$ & Elevé \\
\hline $\begin{array}{l}\text { PROXIMITE } \\
\text { FONCTIONNELLE }\end{array}$ & $\begin{array}{l}\text {-Surface en moyenne de } 1800 \mathrm{~m}^{2} \text { avec des références dans tous les } \\
\text { univers : «Tout sous le même toit ». } \\
\text { •Large choix de services : une plateforme de services pour les } \\
\text { services à domicile/à la personne a été mise en place. }\end{array}$ & Elevé \\
\hline $\begin{array}{c}\text { ProXimite } \\
\text { RELATIONNELLE }\end{array}$ & $\begin{array}{l}\text {-Nombre de salariés élevé, emplois stables. } \\
\bullet \text { Mise en avant d'une « proximité de cour ». } \\
\text { - Carte fidélité donnant droit à de nombreux services (ex : } \\
\text { livraisons à domicile gratuite). } \\
\text {-Application Smartphones « Monoprix et moi ». } \\
\end{array}$ & Elevé \\
\hline $\begin{array}{l}\text { Proximite } \\
\text { IDENTITAIRe }\end{array}$ & $\begin{array}{l}\text { Réel engagement dans le Développement Durable avec : } \\
\text {-Un axe environnemental : mutualisation logistique, véhicule à } \\
\text { gaz naturel et triporteurs à Paris, fluvial, calcul consommation } \\
\text { CO2 des produits. } \\
\text {-Un axe produits bio, produits équitables (Association « Terre de } \\
\text { liens » qui achète des terres agricoles pour les confier à des } \\
\text { agriculteurs fournissant ensuite le groupe), etc. } \\
\text {-Un axe respect et développement des salariés : Académie } \\
\text { Monoprix (formations internes et VAE), Monop'Id : systèmes } \\
\text { d'information pour faire remonter les idées, intégration des } \\
\text { personnes handicapées. }\end{array}$ & Elevé \\
\hline $\begin{array}{l}\text { ProXimite DE } \\
\text { PROCESSUS }\end{array}$ & $\begin{array}{l}\text {-Image de produits de qualité. } \\
\text {-Traçabilité des produits. } \\
\bullet \text { Développement de MDD véhiculant des valeurs : «Inspiration, } \\
\text { qualité, plaisir et accessibilité ». }\end{array}$ & Moyen \\
\hline $\begin{array}{l}\text { PROXIMITE INTER- } \\
\text { ORGANISATIONNELLE }\end{array}$ & $\begin{array}{l}\text {-Proximité surtout en interne au groupe et pas de réel discours sur } \\
\text { les liens avec les fournisseurs. } \\
\text {-Toutefois récemment : développement d'une gamme «petit } \\
\text { producteur », garantie élaborée à moins de 100km du point de } \\
\text { vente. }\end{array}$ & Faible \\
\hline PROXIMITE PRIX & $\begin{array}{l}\text { Positionnement prix élevé, même si modéré par de nombreuses } \\
\text { MDD }\end{array}$ & Faible \\
\hline
\end{tabular}




\section{Tableau 4}

\begin{tabular}{|c|c|c|}
\hline $\begin{array}{l}\text { DIMENSIONS DE LA } \\
\text { PROXIMITE }\end{array}$ & DESCRIPTIF DE CARREFOUR CITY CAFE & $\begin{array}{l}\text { DEGRE DE } \\
\text { PROXIMITE } \\
\text { DANS LE } \\
\text { DISCOURS }\end{array}$ \\
\hline $\begin{array}{l}\text { ProXiMite SPATIALE } \\
\text { OU D'ACCES }\end{array}$ & $\begin{array}{l}\text { Proximité spatiale forte basée sur le choix de zones à fort } \\
\text { passage : gares, université...en hyper centre-ville. Le test de } \\
\text { Jussieu repose essentiellement sur une clientèle étudiante. }\end{array}$ & Elevé \\
\hline $\begin{array}{l}\text { PROXIMITE } \\
\text { FONCTIONNELLE }\end{array}$ & $\begin{array}{l}\text { - Surface de } 100 \text { à } 150 \mathrm{~m}^{2} \text { en libre-service. } \\
\text { - Références concentrées sur le snacking (sandwicherie), la } \\
\text { consommation de café et la presse. } \\
\text { •4 assortiments en rotation pour marquer } 4 \text { moment-clés de la } \\
\text { journée : petit-déjeuner, déjeuner, goûter, dîner. } \\
\text { •large amplitude horaire ( } 7 \mathrm{~h} 30-22 \mathrm{~h} \text { tous les jours), } \\
\text { •quelques services : Wifi, Ipad à disposition pour lecture de la } \\
\text { presse. }\end{array}$ & Elevé \\
\hline $\begin{array}{c}\text { PROXIMITE } \\
\text { RELATIONNELLE }\end{array}$ & •Carte fidélité Carrefour acceptée (pas spécifique au concept). & Faible \\
\hline $\begin{array}{l}\text { ProXimite } \\
\text { IDENTITAIRE }\end{array}$ & $\begin{array}{l}\text { - Café/ Machines Malongo : concept qui se veut une alternative au } \\
\text { café parisien ou à la restauration rapide }\end{array}$ & Moyen \\
\hline $\begin{array}{l}\text { Proximite de } \\
\text { PROCESSUS }\end{array}$ & $\begin{array}{l}\bullet \text { Essentiellement des MDD. } \\
\bullet \text { Notion de fraîcheur des produits : café frais, produits frais à } \\
\text { consommer rapidement. }\end{array}$ & Elevé \\
\hline $\begin{array}{l}\text { PROXIMITE INTER- } \\
\text { ORGANISATIONNELLE }\end{array}$ & $\begin{array}{l}\bullet \text { Filière qualité Carrefour } \\
\text { •Concept en test mais voulu à terme en franchise pour } \\
\text { l'implication dans le tissu local. } \\
\end{array}$ & Faible \\
\hline PROXIMITE PRIX & $\begin{array}{l}\text { Prix bas. Exemple : le produit phare est le petit déjeuner (café, jus } \\
\text { de fruit, croissant) à } 2 \text { euros. }\end{array}$ & Moyen \\
\hline
\end{tabular}




\section{Figure 1}

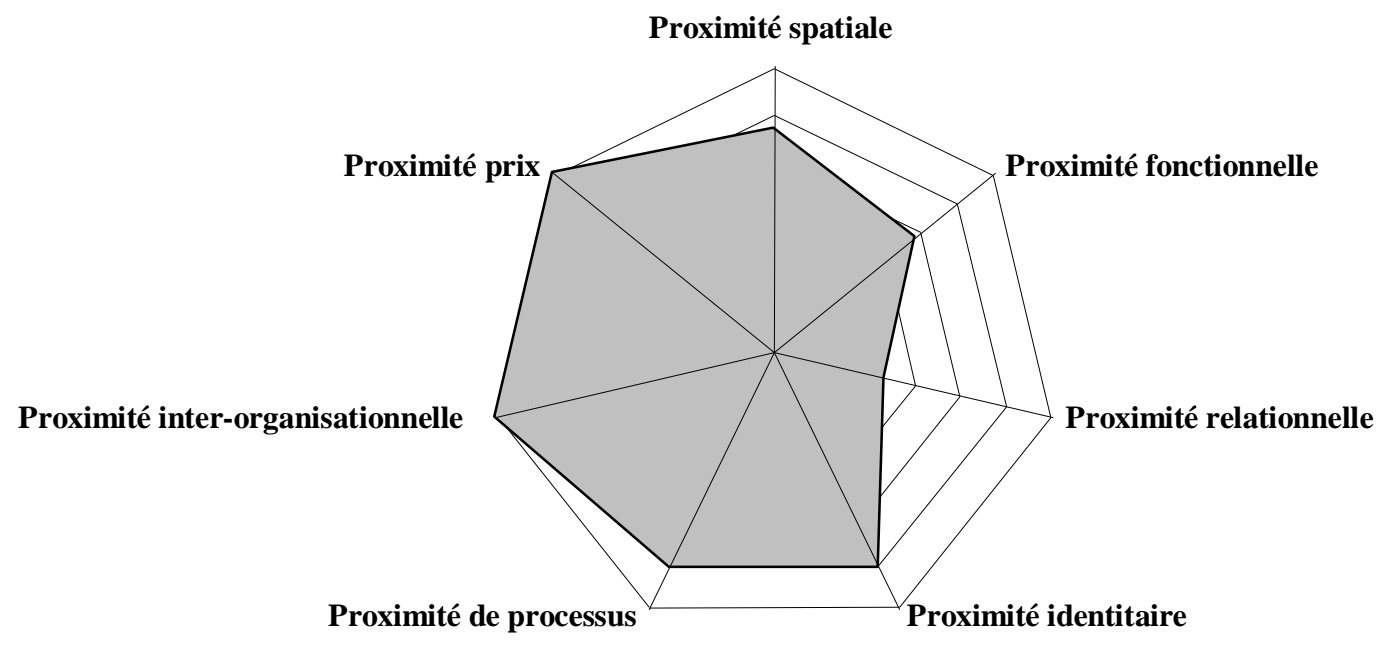




\section{Figure 2}

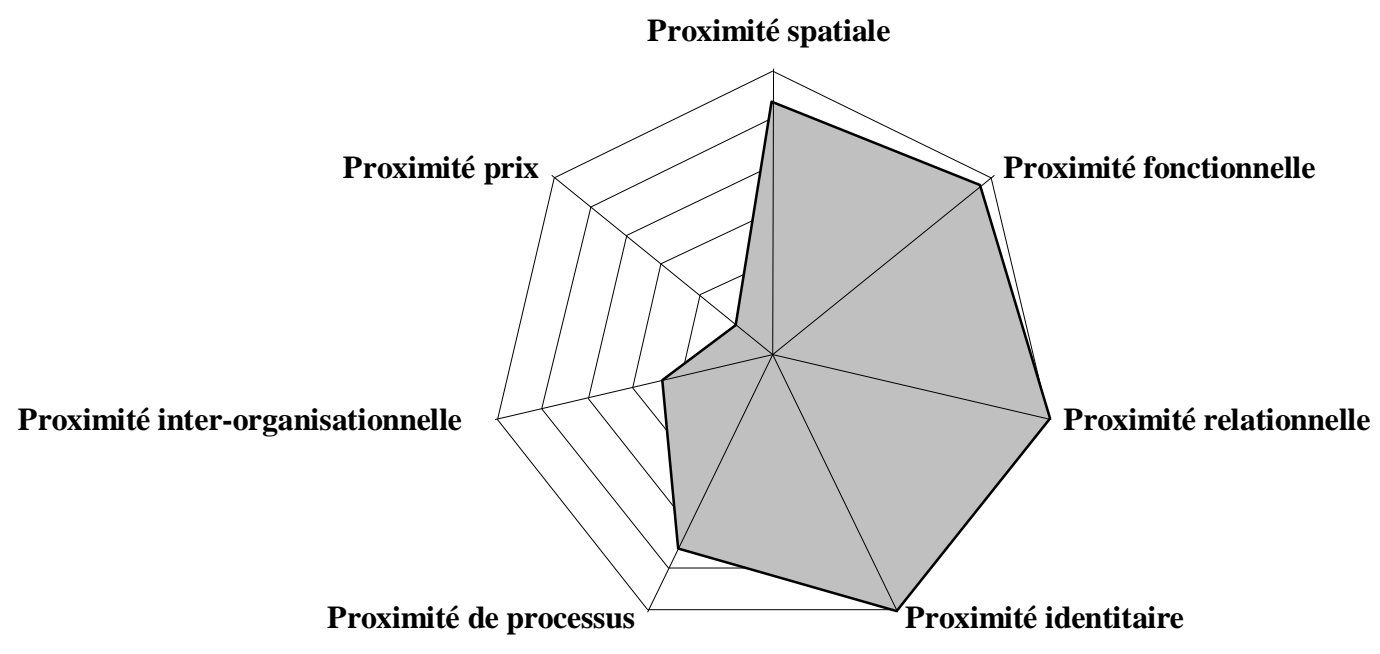




\section{Figure 3}

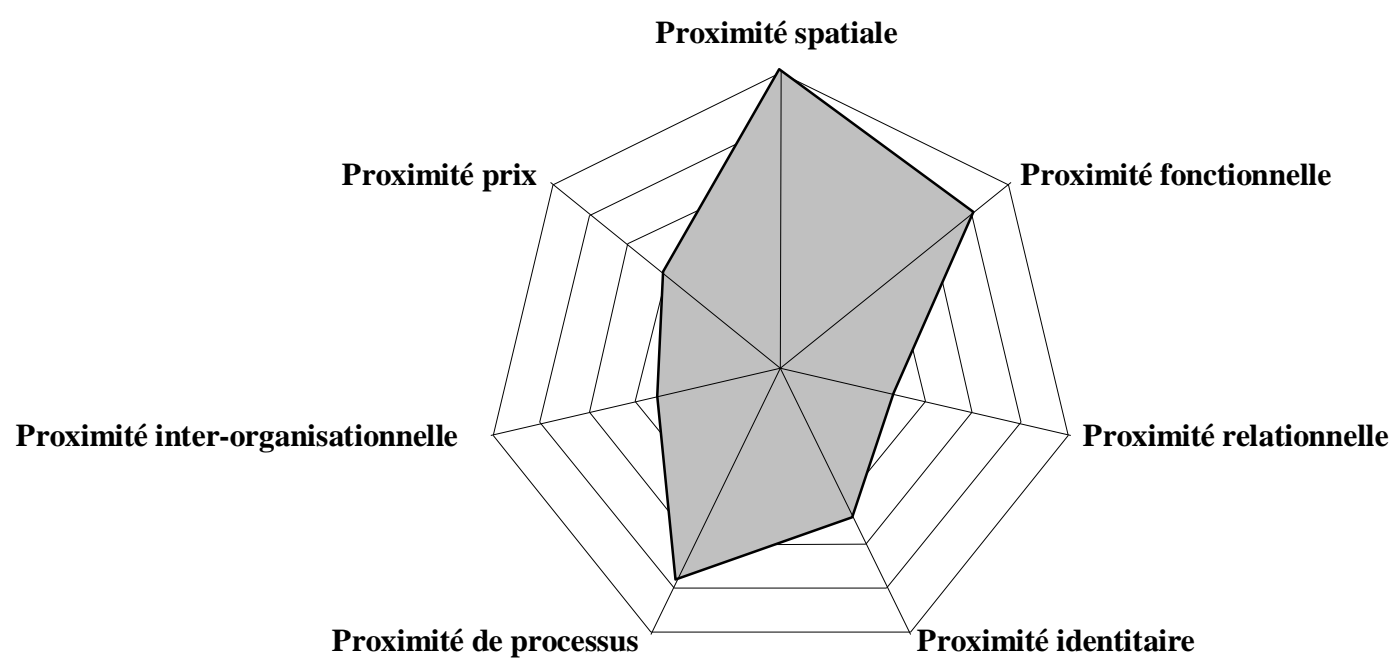


Figure 4

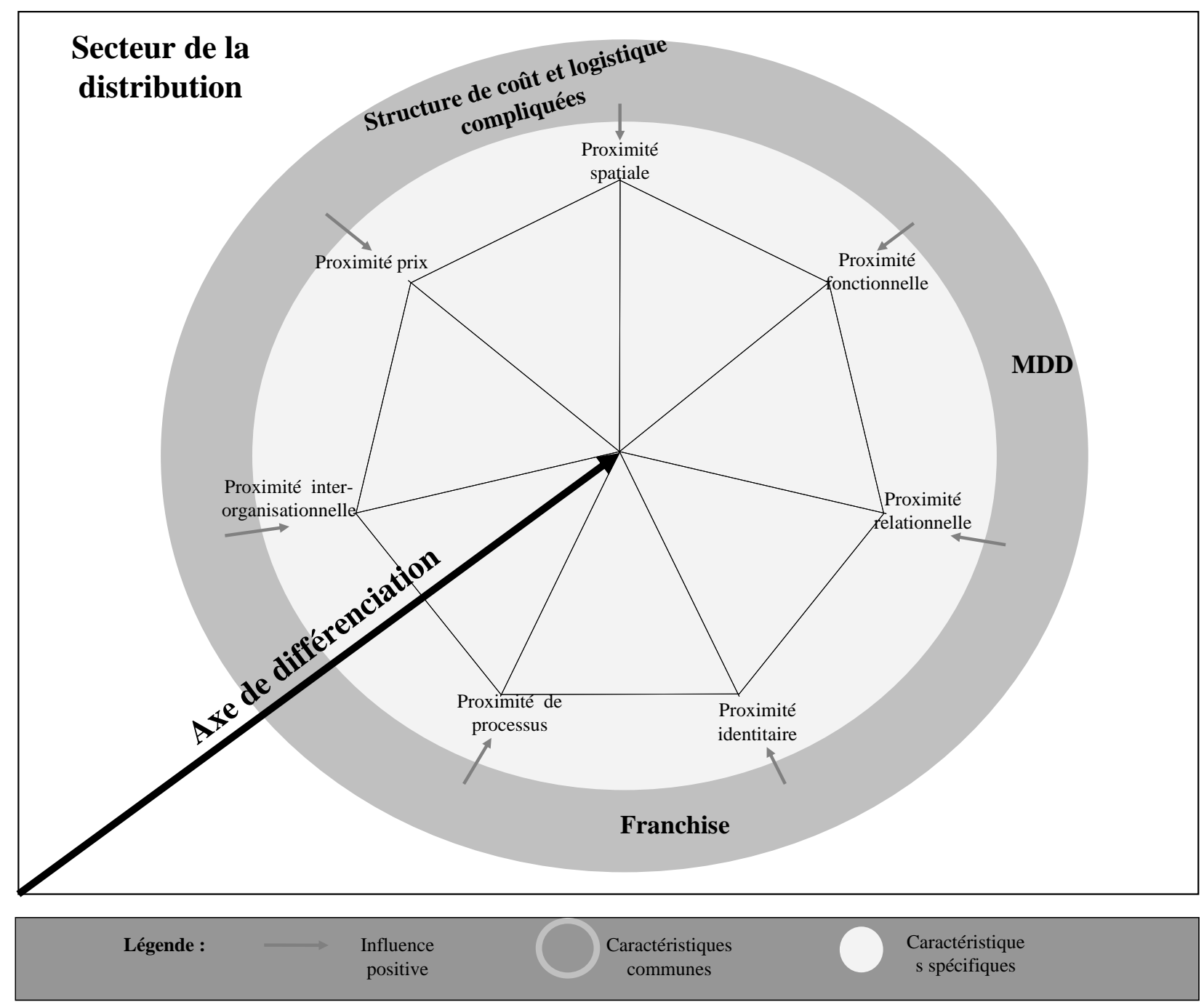

